\title{
CONSTRAINED EQUILIBRIUM POINT OF MAXIMAL MONOTONE OPERATOR VIA VARIATIONAL INEQUALITY
}

\author{
M. PRZEWORSKI and D. ZAGRODNY \\ Received December 4, 1998
}

\begin{abstract}
Herein a sufficient condition for $q$ to belong to $Q \cap T^{-1}(0)$ is provided, where $Q$ is a weakly compact convex subset of a real reflexive Banach space $E$ and $T: E \rightarrow E^{*}$ is a maximal monotone operator.
\end{abstract}

\section{Introduction}

In the paper we are dealing with the problem of finding the constrained equilibrium points of maximal monotone operator i.e. for a given weakly compact convex subset $Q$ of a reflexive Banach space $E$ and $T: E \rightrightarrows E^{*}$ we are looking for a solution of the inclusion $0 \in T(q)$ required to belong to $Q$. Our approach is different than that by the viability method (see [1] for details). We follow S. Simons, who exploring the subdifferential operator of convex function showed that

if for every $\left(x, x^{*}\right) \in$ graph $\partial \psi$ there exists $q \in Q$ such that $<x^{*}, x-q>\geq 0$, then $(Q \times\{0\}) \cap$ graph $\partial \psi \neq \emptyset$, (see $[3,4,5,6])$. He also posed the question whether the implication holds true if we replace the subdifferential by an arbitrary maximal monotone

1991 Mathematics Subject Classification. 49J52, 47H05.

Key words and phrases. Maximal monotonicity, equilibrium point.

ISSN 1425-6908 (c) Heldermann Verlag. 
operator. Herein we give the answer in the affirmative to this question in reflexive Banach space setup. Of course this is a new method ensuring the existence of the constrained equilibrium points for maximal monotone operator in reflexive Banach spaces. The problem still remains open in nonreflexive Banach space as well as the problem of extension of the class of operators beyond the maximal mononotone one.

\section{Basic facts and definitions}

Let $\mathbb{E}$ be a real Banach space with the topological dual $\mathbb{E}^{*}$. For a nonempty convex subset $A$ of $\mathbb{E}$ we define

$$
d_{A}(x)=\inf _{a \in A}\|x-a\|,
$$

where $\|\cdot\|$ is the norm in $\mathbb{E}$. The function $d_{A}^{2}$ is convex and continuous on $\mathbb{E}$.

Below we show that the subdifferential of $d_{A}^{2}$ is singleton on the set $A$, we refer to $[1,2]$ for the definition of the subdifferential of convex function.

Lemma 2.1. Let $A$ be a nonempty convex closed subset of a real reflexive Banach space $\mathbb{E}$. Then

$$
\partial d_{A}^{2}(a)=\{0\}, \text { for every } a \in A \text {. }
$$

Proof. Let $a_{0} \in A$ be fixed. Our proof starts with the observation that

$$
d_{A}^{2}(x) \leq\left\|x-a_{0}\right\|^{2} .
$$

for every $x \in \mathbb{E}$. On the other hand from the convexity and continuity of $d_{A}^{2}$ we get $\partial d_{A}^{2}\left(a_{0}\right) \neq \emptyset$. Let $v^{*} \in \partial d_{A}^{2}\left(a_{0}\right)$, then as a consequence of the definition of the subdifferential of convex function we have

$$
<v^{*}, x-a_{0}>\leq d_{A}^{2}(x)-d_{A}^{2}\left(a_{0}\right)=d_{A}^{2}(x) .
$$

The above inequality and (2.1) imply

$$
<v^{*}, x-a_{0}>\leq\left\|x-a_{0}\right\|^{2} .
$$

Thus $v^{*} \in \partial\left\|\cdot-a_{0}\right\|^{2}\left(a_{0}\right)$, so the following implication holds true

$$
v^{*} \in \partial d_{A}^{2}\left(a_{0}\right) \Longrightarrow v^{*} \in \partial\left\|\cdot-a_{0}\right\|^{2}\left(a_{0}\right) \text {. }
$$

Of course, we have also

$$
\partial\left\|\cdot-a_{0}\right\|^{2}\left(a_{0}\right)=\partial\|\cdot\|^{2}(0)=\{0\},
$$

so $v^{*}=0$. This completes the proof. 
Now we recall some basic facts about the maximal monotone operators. Let $S: \mathbb{E} \rightrightarrows \mathbb{E}^{*}$ be a maximal monotone operator, we denote the effective domain of $S$ by dom $S$ i.e.

$$
\operatorname{dom} S:=\{x \in \mathbb{E} \mid \mathbb{S}(\curvearrowleft) \neq \emptyset\}
$$

and by $R(S)$ we denote the range of $S$ i.e.

$$
R(S):=\bigcup_{x \in \operatorname{dom} S} S(x)
$$

The graph of $S$ is the set

$$
\text { graph } S:=\left\{\left(x, x^{*}\right) \in \mathbb{E} \times \mathbb{E}^{*} \mid \curvearrowleft^{*} \in \mathbb{S}(\curvearrowleft)\right\} .
$$

Let us recall the following definition of weakly coercive multimapping (see [7], Definition 32.34).

Definition 2.2. Let $S: \mathbb{E} \rightrightarrows \mathbb{E}^{*}$. $S$ is called weakly coercive iff either dom $S$ is bounded or dom $S$ is unbounded and

$$
\inf _{x^{*} \in S(x)}\left\|x^{*}\right\| \longrightarrow+\infty \text { as }\|x\| \longrightarrow+\infty, x \in \operatorname{dom} S
$$

Ending this section we recall some known fact concerning weakly coercive operators (see [7], Corollary 32.35).

Theorem 2.3. Let $S: \mathbb{E} \rightrightarrows \mathbb{E}^{*}$ be a maximal monotone and weakly coercive operator on a real reflexive Banach space $\mathbb{E}$. Then $R(S)=\mathbb{E}^{*}$.

\section{Main result}

In this section we provide an answer to Simons' question, concerning the maximal monotone operators on a reflexive Banach space.

We start with the following lemma.

Lemma 3.1. Let $\mathbb{E}$ be a real reflexive Banach space, $T: \mathbb{E} \rightrightarrows \mathbb{E}^{*}$ be a maximal monotone operator with $\operatorname{dom} T \neq \emptyset$. Let $Q$ be a nonempty convex closed and bounded subset of $\mathbb{E}$. Then $R\left(T+\partial d_{Q}^{2}\right)=\mathbb{E}^{*}$.

Proof. It follows from the definition of $d_{Q}^{2}$ that

$$
\operatorname{dom} T \cap \operatorname{int}\left(\operatorname{dom} \partial d_{Q}^{2}\right) \neq \emptyset,
$$

so by the classical Rockafellar theorem $T+\partial d_{Q}^{2}$ is maximal monotone (see [7], Theorem 32.I) and of course

$$
\operatorname{dom}\left(T+\partial d_{Q}^{2}\right)=\operatorname{dom} T \text {. }
$$


Because of Theorem 2.3 it is enough to prove that operator $T+\partial d_{Q}^{2}$ is weakly coercive. We observe that if dom $T$ is bounded then the weak coercivity of $T+\partial d_{Q}^{2}$ follows immediately from Definition 2.2.

Let us consider the case when $\operatorname{dom} T$ is unbounded. Let $\left\{z_{n}\right\}_{n=1}^{\infty} \subset$ dom $T$ be such that

$$
\left\|z_{n}\right\| \longrightarrow+\infty \text { as } n \longrightarrow+\infty \text { and } z_{n}^{*} \in T\left(z_{n}\right) .
$$

Without loss of generality we can assume that $0 \in T(0)$. If not we can translate $\operatorname{dom} T$ and $R(T)$ (keep in mind that $d_{Q}^{2}(x+y)=d_{Q-y}^{2}(x)$ ). Now the above assumptions imply

$$
<z_{n}^{*}, z_{n}>\geq 0 \text { for every } n \in \mathbb{N} \text {. }
$$

Let $x_{n}^{*} \in \partial d_{Q}^{2}\left(z_{n}\right)$, then we get

$$
<x_{n}^{*}, 0-z_{n}>\leq d_{Q}^{2}(0)-d_{Q}^{2}\left(z_{n}\right),
$$

so

$$
<x_{n}^{*}, z_{n}>\geq d_{Q}^{2}\left(z_{n}\right)-d_{Q}^{2}(0) .
$$

Let us choose $r>0$ such that $Q \subset B(0, r)$. For sufficiently large $n$ we have $\left\|z_{n}\right\|>r$ and

$$
d_{Q}^{2}\left(z_{n}\right) \geq d_{B(0, r)}^{2}\left(z_{n}\right)=\left(\left\|z_{n}\right\|-r\right)^{2}>0 .
$$

From the last inequality and by (3.2) we obtain

$$
<x_{n}^{*}, z_{n}>\geq\left\|z_{n}\right\|^{2}-2 r\left\|z_{n}\right\|+r^{2}-d_{Q}^{2}(0) \text { for sufficiently large } n \text {. }
$$

It follows from (3.1) and (3.3) that

So

$$
<x_{n}^{*}+z_{n}^{*}, \frac{z_{n}}{\left\|z_{n}\right\|}>\geq\left\|z_{n}\right\|-2 r+\frac{r^{2}-d_{Q}^{2}(0)}{\left\|z_{n}\right\|},
$$

$$
\left\|z_{n}\right\|-2 r+\frac{r^{2}-d_{Q}^{2}(0)}{\left\|z_{n}\right\|} \leq\left\|x_{n}^{*}+z_{n}^{*}\right\| .
$$

for every $z_{n}^{*} \in T\left(z_{n}\right)$ and $x_{n}^{*} \in \partial d_{Q}^{2}\left(z_{n}\right)$. This inequality we can rewrite as

$$
\left\|z_{n}\right\|-2 r+\frac{r^{2}-d_{Q}^{2}(0)}{\left\|z_{n}\right\|} \leq \inf _{x^{*}+z^{*} \in\left(T+\partial d_{Q}^{2}\right)\left(z_{n}\right)}\left\|x^{*}+z^{*}\right\|
$$

and passing to the limit with $n \longrightarrow+\infty$ we get

$$
\inf _{x^{*}+z^{*} \in\left(T+\partial d_{Q}^{2}\right)(x)}\left\|x^{*}+z^{*}\right\|=+\infty \text { as }\|x\| \longrightarrow+\infty, x \in \operatorname{dom} T .
$$

This means that $T+\partial d_{Q}^{2}$ is weakly coercive operator. By Theorem 2.3 we have $R\left(T+\partial d_{Q}^{2}\right)=\mathbb{E}^{*}$ and the proof is complete. 
Now we are ready to prove our main result in this paper, which is the solution to the Simons' problem in refexive Banach space setup (see [3]).

Theorem 3.2. Let $Q$ be a nonempty convex weakly compact subset of a real reflexive Banach space $E$. Let $T: \mathbb{E} \rightrightarrows \mathbb{E}^{*}$ be a maximal monotone operator with dom $T \neq \emptyset$, which satisfies the following condition

for every $\left(x, x^{*}\right) \in \operatorname{graph} T$ there exists $q \in Q$

$$
\text { such that }\left\langle x^{*}, x-q>\geq 0\right. \text {. }
$$

Then

$$
(Q \times\{0\}) \cap \text { graph } T \neq \emptyset .
$$

Proof. Let us define the maximal monotone operator $T_{1}:=T+\partial d_{Q}^{2}$ (see the Rockafellar theorem [7]). It is easy to see that $T_{1}$ fulfills the following condition

for every $\left(x, x^{*}\right) \in \operatorname{graph} T_{1}$ there exists $q \in Q$

$$
\text { such that }<x^{*}, x-q>\geq d_{Q}^{2}(x) \text {. }
$$

By Lemma 3.1 we obtain $R\left(T_{1}\right)=\mathbb{E}^{*}$ so $0 \in R\left(T_{1}\right)$. Let $0 \in T_{1}\left(x_{0}\right)$. Hence there are $t_{0}^{*} \in T\left(x_{0}\right)$ and $x_{0}^{*} \in \partial d_{Q}^{2}\left(x_{0}\right)$ for which $0=t_{0}^{*}+x_{0}^{*}$. By (3.6) we can find $q_{0} \in Q$ such that

$$
0=<0, x_{0}-q_{0}>=<t_{0}^{*}+x_{0}^{*}, x_{0}-q_{0}>\geq d_{Q}^{2}\left(x_{0}\right),
$$

which gives $x_{0} \in Q$. Lemma 2.1 forces that $x_{0}^{*}=0$ so $t_{0}^{*}=0$, which completes the proof.

\section{References}

[1] Aubin, J.-P. and Frankowska, H., Set-Valued Analysis , Birkhauser, Boston, 1990.

[2] Clarke, F.H., Optimization and Nonsmooth Analysis, Jonh Wiley, New York, 1983.

[3] Simons, S., Subtangents with controlled slope, Nonlinear Anal. 22 (1994), 1373-1389.

[4] Simons, S., Swimming below icebergs, Set-Valued Anal. 2 (1994), 327-337.

[5] Simons, S., Minimax and Monotonicity, Lecture Notes in Math. 1693, SpringerVerlag, New York, 1998.

[6] Zagrodny, D., The maximal monotonicity of the subdifferentials of convex functions: Simons' problem, Set-Valued Anal. 4 (1996), 301-314.

[7] Zeidler, E., Nonlinear Functional Analysis and Its Applications II: Monotone Operators, Springer-Verlag, New York, Berlin, 1986. 
Miroseaw Przeworski

Institute OF MATHEMATICS

TECHNICAL UNIVERSITY OF RADOM

MALCZEWSKIEGO 20A

26-600 RAdOM, POLAND

MPRZEWOR@KIUX.MAN.RADOM.PL
DARIUSZ ZAGRODNY INSTITUTE OF MATHEMATICS TECHNICAL UNIVERSITY OF ŁÓDŹ

ŻWIRKI 36

90-924 Łódź, POLAND ZAGRODNY@CK-SG.P.LODZ.PL 